\title{
Auswirkung des muskuläre Dysbalances zwischen Plantar- und Dorsalflexoren auf die Verletzungen von Badminton Spieler
}

\author{
Walaa eldin A. Hazzaah \\ Fakultät für Leibeserziehung, AlHaram, Universität Helwan, Ägypten
}

\section{Zusammenfassung}

\section{Hintergrund:}

muskuläre Dysbalance führt zu instabilität des Sprunggelenkes für Badminton Spieler und das Risiko für Spielverletzungen steigern. Die Studie verfolgt das Ziel, die Auswirkung des muskuläre Dysbalances der Plantar- und Dorsalflexoren auf die Verletzungen von Badminton Spieler zu klären.

\section{Methoden:}

Auf dem Isomed 2000 mit Messwiederholungstest nahmen 33 männliche Badminton Spieler $(\mathrm{KH}=$ $184,4 \pm 7,0 \mathrm{~cm}, \mathrm{KM}=84,6 \pm 11 \mathrm{~kg}$, Alter $=24,2 \pm 6,0 \mathrm{~J}$.) teil, die zwei bis drei Mal wöchentlich Badminton Spielen.

Ergebnisse: Wie hypothetisch angenommen, zeigte sich eine muskuläre Dysbalance des Sprunggelenks, hervorgerufen durch ein lokales Ermüdungsprotokoll mit gleicher Wiederholungszahl, welches die muskuläre Leistungsfähigkeit der Dorsalflexoren stärker herabsetzte als die der Plantarflexoren, was auf ein erhöhtes Verletzungsrisiko nach Ermüdung hinweist. Um das Verletzungsrisiko zu vermindern wird empfohlen beim Krafttraining besonders die weniger leistungsfähigen Dorsalflexoren aufmerksamer zu beachten.

\section{Schlussfolgerung:}

Zur Schtzung des Sprunggelenkes gegen Verletzungen sollte ergänzend Kraft Einheiten geplant in dem Trainingsprogramm der Fußmuskulatur mit einem besonderen Schwerpunkt auf die weniger leistungsfähigen Muskulatur ergänzend zum Training Einheiten integriert werden.

\section{Schlüsselworter: Isokinetik, Isometrik, Isomed2000 Einleitung}

Muskelermüdung wird als ursächlicher Faktor für viele Verletzungen angenommen (GarcíaPérez, Pérez-Soriano, Llana, Martínez-Nova, \& Sánchez-Zuriaga, 2013; Kellis \& Liassou, 2009). Dabei betreffen über 90 Prozent die untere Extremität, wovon ca. ein Drittel jeweils auf das Knie, den Unterschenkel und den Fuß entfallen (Fredericson \& Misra, 2007; Hreljac, 2005).

Beim Badminton Spielen trägt der Fuß das 2 bis 3-fache des Körpergewichts. Damit diese Kräfte den passiven Bewegungsapparat nicht über- oder fehlbelasten, muss die Muskulatur der unteren Extremität für eine adäquate Schockabsorption sorgen, beispielsweise durch exzentrische Kontraktion der Plantarflexoren (Noakes, 1991). Ermüdet die Muskulatur jedoch, ist eine ausreichende Absorption der Aufprallkräfte nicht mehr gewährleistet, der passive Bewegungsapparat wird in Mitleidenschaft gezogen und das Risiko für Laufverletzungen steigt. Dieser Zusammenhang zwischen muskulärer Ermüdung und Verletzungsrisiko wurde bereits von verschiedenen Studien (Baker, Burnstein und Franke, 1972; Burr, 1997; Dickinson, Cook, \& Leinhardt, 1985; Grimston \& Zernike, 1993; Nyland, Shapiro, Stine, Horn, \& Ireland, 1994) gezeigt. Wie genau sich der Landung des Fußes in Folge der Ermüdung verändert, konnte trotz zahlreicher Studien bisher allerdings nicht hinreichend geklärt werden. Um verletzungsprophylaktische Trainingshinweise generieren zu können, muss bekannt sein, wie sich der Landung des Fußes durch Ermüdung im Detail verändert. Zumal Badminton Spieler /innen mit Vorverletzungen der unteren Extremität ein erhöhtes Risiko für Folgeverletzungen haben (Macera, 1992; Marti, Vader, Minder, \& Abelin, 1988; Walter, Hart, McIntosh, \& Sutton, 1989). Insofern sollte im Idealfall bereits eine erste Verletzung verhindert werden.

Obwohl die Anzahl von Veröffentlichungen zum Einfluss muskulärer Ermüdung auf die plantare Druckverteilung und die Bodenreaktionskräfte beim Laufen seit 2006 deutlich ansteigt, kommen die Studien zu unterschiedlichen Ergebnissen und der Effekt der Ermüdung ist noch nicht abschließend geklärt. In einer Metaanalyse zur Veränderung der Bodenreaktionskraft nach Ermüdung stellten Zadpoor und Nikooyan (2012) fest, dass die aktive Spitze der vertikalen Bodenreaktionskraft 
bei den meisten Untersuchungen im Mittelpunkt steht, weil sie die muskuläre Reaktion bezüglich des Aufpralls auf dem Untergrund widerspiegelt. Dabei dominieren zwei Theorien. Einerseits wird angenommen, dass die Fähigkeit der adäquaten Schockabsorption bei Ermüdung abnimmt, woraufhin die Bodenreaktionskraft ansteigt, um diesen Effekt entgegenzuwirken. Andererseits wird eine Verringerung der Bodenreaktionskraft unterstellt, da der menschliche Körper über eine Schutzstrategie verfügt, die diese Reaktion veranlasst, um Verletzungen vorzubeugen (Zadpoor \& Nikooyan, 2012).

Die Metaanalyse schloss acht Studien ein, die den aktive Spitze der Bodenreaktionskraft mittels Kraftmessplatten vor und nach Ermüdung durch Laufen ermittelten. Drei Studien zeigten eine signifikante Abnahme (Girard, Millet, \& Slawinski, 2010; Nummela, Rusko, \& Mero, 1994; Rabita, Slawinski, Girard, Bignet, \& Hausswirth, 2010), wohingegen vier Studien nur geringe nicht signifikante Veränderungen feststellten (Dickinson et al., 1985; Gerlach, White, \& Burton, 2005; Nyland et al., 1994; Slawinski, Heubert, \& Quievre, 2008). Eine weitere Studie untersuchte die Bodenreaktionskraft beim Laufen nach lokaler Muskelermüdung der Dorsalflexoren und -invertoren des Fußes (Christina, White, \& Gilchrist 2001), wobei die Dorsalflexion zu einem nicht signifikanten Anstieg und die Inversion zu einer Verringerung der Bodenreaktionskraft führte.

Andere Studien zur Bodenreaktionskraft kamen ebenfalls zu widersprüchlichen Befunden. Nach extremer Ermüdung durch einen 24-Stunden-Laufband bzw. Ultrabergmarathon registrierten Morin, Samozino und Millet (2011) bzw. Morin, Tomazin, Edouard und Millet (2011) eine signifikante Abnahme der Bodenreaktionskraft vom 2,24 fache auf das 2,14 fache bzw. vom 2,32 fache auf das 2,17 fache des Körpergewichts. Quammen et al. (2012) verglichen zwei unterschiedliche Ermüdungsprotokolle miteinander, wobei sowohl Krafttests und Sprints als auch die Krafttests und ein 30minütiger Laufbandlauf zu einem nicht signifikanten Anstieg der Bodenreaktionskraft führten.

Zur plantaren Druckverteilung liegen ebenfalls kontroverse Befunde nach ermüdendem Laufen über unterschiedliche Distanzen (10-km- bis Marathonlaif bzw. 30-min-Lauf) vor. Einige Studien fanden einen Anstieg der Vorfußbelastung nach Laufermüdung (Bisiaux \& Moretto, 2008; Willems, Ridder, \& Roosen, 2012; Willson \& Kernozek, 1999) und nach lokaler Ermüdung der Plantar- und Dorsalflexoren (Olivier, 2013). Inkonsistenzen wurden zur Wirkung der Ermüdung durch Laufen auf die Druckverteilung unter der Ferse, dem Mittelfuß und den Zehen referiert. Unter der Ferse wurde sowohl über einen signifikanten Anstieg (Weist, Eils, \& Rosenbaum, 2004; Willems et al., 2012), als auch Abfall des Spitzendruckes (Alfuth \& Rosenbaum, 2011; Bisiaux \& Moretto, 2008; Garcia-Perez et al., 2013; Willson \& Kernozek, 1999) berichtet. Einige Studien beobachteten eine Zunahme unter den Metatarsalen (Bisiaux \& Moretto, 2008; Nagel, Fernholz, Kibele, \& Rosenbaum, 2008; Weist et al., 2004; Willems et al., 2012; Willson \& Kernozek, 1999) und unter dem medialen Mittelfuß, (Weist et al., 2004, Willems et al., 2012), aber andere zeigten dagegen einen Abfall unter dem lateralen bzw. medialen Mittelfuß (Alfuth \& Rosenbaum, 2011; Bisiaux \& Moretto, 2008). Zu den Druckwerten unter den Zehen wurden eine signifikante Reduktion (Garcia-Perez et al., 2013; Nagel et al., 2008, Olivier, 2013; Willems et al., 2012; Willson \& Kernozek, 1999), aber auch in zwei weiteren Studien keine Unterschiede (Bisiaux \& Moretto, 2008; Weist et al., 2004) gefunden.

Die Ermüdung beeinflusst die Schrittfrequenz und Schrittlänge beim Badminton Spielen, wobei sich eine Reduktion der Schrittfrequenz und ein Anstieg der Schrittlänge (Garcia-Perez et al., 2013; Gerlach et al., 2005), eine geringere (Hunter \& Smith, 2007) bzw. kein Wechsel (Alfuth \& Rosenbaum, 2011) in der Schrittfrequenz zeigten. Außerdem zeichnet sich Laufen auf dem Laufband durch eine veränderte Schrittfrequenz aus (Garcia-Perez et al., 2013; Riley et al., 2008; Schache et al., 2001; Wank, Frick, \& Schmidtbleicher, 1998). Bei moderater Laufgeschwindigkeit auf dem Laufband wurden eine kürzere Schrittlänge und eine höhere Schrittfrequenz im Vergleich zum Laufen auf natürlichem Untergrund beobachtet (Hong, Wang, Li, \& Zhou, 2012). Aufgrund der Beeinflussung der Laufbewegung werden Laufbandergebnisse kontrovers diskutiert (Garcia-Perez et al., 2013), aber von verschiedenen Autoren trotzdem als repräsentativ für Laufuntersuchungen betrachtet (Fellin, Manal, \& Davis, 2010; Riley et al., 2008; Schache et al., 2001).

Im Allgemeinen ist die Kraftfähigkeit beider Beine nicht gleich, wobei das Sprungbein kräftiger ist als das Spielbein. Deshalb wird unterschieden zwischen dominantem Bein, das im Sprungtest höhere Weiten erzielt und dem bevorzugten Bein, das zur Manipulierung von Gegenständen (z.B. einen Fußball) genutzt wird (Van der Harst, Gokeler, \& Hof, 2007). Zur Beinasymmetrie beim Laufen existieren nur wenige biomechanische Analysen, die keine signifikanten Differenzen in der Kinematik 
und der Bodenreaktionskraft im Vergleich des dominanten vs. nicht-dominanten (bevorzugten) Beines vor bzw. nach Ermüdung (Brown, Zifchock, \& Hillstrom, 2013; Hamil, Bates, \& Knutzen, 1984) ergaben.

Die Befunde zum Einfluss der Ermüdung auf die plantare Druckverteilung beim Badminton Spielen sind somit aufgrund der unterschiedlichen Ermüdungsprotokolle und der abweichenden Fußzonen widersprüchlich. Außerdem fehlt häufig die Berücksichtigung der Fußaufsatztechnik, der Beindominanz, verschiedener Laufgeschwindigkeiten und/ oder der Messwiederholung. Die Mehrzahl der Studien verwendete erschöpfendes Laufen (García-Pérez, 2013; Zadpoor \& Nikooyan, 2012) ohne differenzieren zu können, welche Veränderungen direktes Resultat der lokalen Muskelermüdung darstellen. Elektromyografische Untersuchungen der Dorsal- und Plantarflexoren des Fußes belegen, dass diese Muskeln zwischen 50 bis $85 \%$ des Laufzyklus aktiv sind und somit stark ermüden können (Kellis \& Liassou, 2009; Reber, Perry, \& Pink, 1993). Dabei stellte sich beim Laufen mit progredienter Ermüdung eine muskuläre Dysbalance ein, da die Aktivität der Plantarflexoren konstant blieb, während die Aktivität der Dorsalflexoren abfiel (Mizrahi, Verbitsky, Isakov, \& Daily, 2000).

Mit der Studie wird das Ziel verfolgt, den Einfluss eines standardisierten Ermüdungsprotokolls der Plantar- und Dorsalflexoren auf die plantare Druckverteilung unter drei Fußzonen beim Laufen in drei Geschwindigkeiten zu untersuchen und dabei mögliche Testeffekte sowie die Beinasymmetrie als konfundierende Variablen zu berücksichtigen.

Hypothetisch wird angenommen, dass ein lokales Ermüdungsprotokoll zu einer unterschiedlich herabgesetzten muskulären Leistungsfähigkeit der Plantar- und Dorsalflexoren führt und sich im anschließenden Laufen mit verschiedenen Geschwindigkeiten in vergleichbaren Veränderungen der plantaren Druckverteilung unter dem Fuß niederschlägt.

Material und Methoden

\section{Studiendesign und Stichprobe}

Diese Laborstudie umfasste eine Übungseinheit mit anschließender Messwiederholung im PräPostdesign innerhalb von drei bis sieben Tagen, um eine vollständige Regeneration der Muskeln zu gewährleisten. In der Übungseinheit wurden die Testabläufe festgelegt und die Einstellung der Geräte an den jeweiligen Probanden bestimmt.

An der Studie nahmen 33 männliche Badminton Spieler $((\mathrm{KH}=184,4 \pm 7,0 \mathrm{~cm}, \mathrm{KM}=84,6 \pm 11 \mathrm{~kg}$, Alter $=24,2 \pm 6,0 \mathrm{~J}$.) teil, die zwei bis drei Mal wöchentlich Badminton Spielen. Zur Teilnahme wurden ausschließlich Badminton Spieler ausgewählt. Die Testabläufe wurden durchgeführt an dem Kraftmessgerät IsoMed 2000 Dynamometer (D\&R FERSTL GmbH, Germany) und dem Laufband Typ: h/p/cosmos quasar -FDM-THQ-M der Firma Zebris Medical GmbH,Germany.Beide Geräte entsprechen den medizinischen Sicherheitsstandards.Die Kalibrierung der Geräte war zertifitiert und wurde am Testtag überprüft.

\section{Untersuchungsablauf}

Nach einer Erwärmung von 10 Minuten mit steigender Laufgeschwindigkeit bis maximal $15 \mathrm{~km} / \mathrm{h}$ startete der Laufbandtest ohne Vorermüdung (Baseline) mit variierter Laufgeschwindigkeit in den Stufen 11,13 und $15 \mathrm{~km} / \mathrm{h}$. Die Badminton Spieler liefen mit Socken ohne Schuhe, um den Einfluss des Sportschuhs zu eliminieren. Nach einer kurzen Gewöhnung an die jeweilige Laufgeschwindigkeit zeichnete das Messsystem Laufzyklen über 30 s auf. Daran schloss sich das standardisierte Ermüdungsprotokoll für das erste Bein (Krafttest). Dazu absolvierte der Proband zunächst den isometrischen Maximalkrafttest, der drei Sätze a einer maximalen Kontraktion von $5 \mathrm{~s}$ und einer Satzpause von 3 Minuten umfasste. Die erste Serie diente als Probe, die beiden anderen als Test. Der Winkel im Sprunggelenk betrug jeweils $90^{\circ}$.

\section{Testbeschreibung}

Begonnen wurde mit der Plantarflexion. Das anschließende isokinetische Ermüdungsprotokoll setzte sich aus zehn Sätzen a sechs Wiederholungen konzentrischer Kontraktionen der Plantar- und Dorsalflexoren mit $10 \mathrm{~s}$ Satzpause und einer Winkelgeschwindigkeit von $60 \%$ susammen. Das Bewegungsausma $\beta$ betrug maximal $55^{\circ}$ für die Plantarflexion und $25^{\circ}$ für die Dorsalflexion. Begonnen wurde mit der Plantarflexion. Im Anschluss erfolgte die Laufanalyse nach Vorermüdung dieses Beines. Der Ablauf erfolgte wie ohne Vorermüdung. An dem Kraftmessgerät wurde der isometrische Maximalkrafttest und isokinetische Ermüdungsprotokoll an dem zweiten Bein 
durchgeführt. Die Reihenfolge der Vorermüdung des Beines (links versus rechts) sowie der Laufgeschwindigkeiten erfolgte randomisiert. Abschließend wurde die Laufanalyse nach Vorermüdung für das zweite Bein durchgeführt.

\section{Mathematisch-statistische Auswertung}

Die Datenauswertung der Krafttests erfolgte über die Herstellersoftware (IsoMed Analyse V.1.0.5). Für den isometrischen Test wurde das maximale Drehmoment aus beiden Versuchen und für den isokinetischen Test die Mittelwerte und Standardabweichungen der maximalen Drehmomente der 60 Wiederholungen ermittelt. Der Ermüdungsindex wurde als Koeffizient des mittleren Drehmoments aus dem isokinetischen Ermüdungsprotokoll durch das maximale Drehmoment aus dem isometrischen Maximalkrafttest gebildet. Ein kleiner Koeffizient wies somit auf eine starke lokale Muskelermüdung hin. Die Reproduzierbarkeit der Kraftdaten war gut bis exzellent. Die relative Reproduzierbarkeit erreichte Intraklassenkorrelationswerte (ICC) für die Plantar- bzw. Dorsalflexoren beim isometrischen Maximalkrafttest von 0,96-0,99 bzw. 0,90-0,98, beim Ermüdungsprotokoll von 0,89-0,97 bzw. 0,870,97 sowie beim Ermüdungsindex von 0,84-0,96 bzw. 0,76-0,94. Die absolute Reproduzierbarkeit lag für die Plantar- bzw. Dorsalflexoren beim isometrischen Maximalkrafttest bei 3,1 bzw. 6,4 \%, beim Ermüdungsprotokoll bei 6,2 bzw. 10,7 \% sowie beim Ermüdungsindex bei 12,3 bzw. 21,8 \%

Die Auswertung der Laufbanddaten erfolgte mit der Software FDM-T Version 0.39 der zebris Medical GmbH (2009). Standardmäßig wurden Schrittlänge und -frequenz sowie die gemittelten maximalen plantaren Druckwerte getrennt für die drei Fußzonen Ferse, Mittelfuß und Vorfuß sowie das erste passive Kraftmaximum aus den gemittelten maximalen Druckwerten der Laufzyklen über 30 s berechnet (Tab. 3 und 4).

Die Daten wurden zunächst als arithmetische Mittelwerte und Standardabweichungen beschrieben. Der Einfluss der lokalen Muskelermüdung, der Testwiederholung und der Beinasymmetrie sowie der Laufgeschwindigkeit auf die Druckverteilung, Schrittfrequenz und Schrittlänge beim Laufen wurde mittels Varianzanalysen mit Messwiederholung nach dem allgemeinen linearen Modell geprüft. Die Normalverteilung und Varianzhomogenität wurden mit dem Kolmogorov-Smirnov-Test und mit dem Levene-Test begutachtet. Als Kennwert der Effektstärke diente das partielle Eta-Quadrat (np2), wobei folgende Klassifizierung festgelegt wurde: kleiner Effekt, wenn $\eta p 2=0,08$, mittlerer Effekt, wenn $\eta p 2=0,20$ und großer Effekt, wenn $\eta p 2=0,32$ (Cohen, 1992).Der LSD-Test (Least Significant Difference) prüfte die Signifikanz der paarweisen Vergleiche. Ein $\alpha$-Niveau von $p \leq 0,05$ wurde als signifikantes Ergebnis eingestuft. Die Datenverarbeitung erfolgte mittels des statistischen Computerprogramms IBM SPSS 20.0 (Chicago, IL, USA).

\section{Ergebnisse}

Tabelle 1 stellt die signifikanten Effekte und die Tabellen 2, 3 und 4 die Mittelwerte und Standardabweichungen sowie die Signifikanzen der paarweisen Vergleiche dar. Das Kraftprotokoll führte zu einer lokalen Muskelermüdung mit signifikanten Unterschieden zwischen dem linken und rechten Bein mit hoher Effektstärke. Ein signifikanter Effekt der Messwiederholung bestand dabei nicht (Tab. 1). Höhere Werte wurden für das linke Bein bei der Plantar- und Dorsalflexion sowohl für die Maximalkraft als auch für das Ermüdungsprotokoll im Test 1 und Test 2 gefunden. Infolge des Ermüdungsprotokolls sanken die Plantarflexoren auf $52-62 \%$ und die Dorsalflexoren auf $35-41 \%$ der isometrischen Maximalkraft. Der Ermüdungskoeffizient wies eine stärkere lokale Muskelermüdung der Plantarflexoren des linken Beines auf. Dagegen wurde bei den Dorsalflexoren das rechte Bein stärker ermüdet (Tab. 2). 
Tabelle 1: Kennwerte der Varianzanalyse Krafttest (einfaktoriell) und Laufbandtest (Druckmaxima dreifaktoriell und Schrittfrequenz und Doppelschrittlänge zweifaktoriell), degree of freedom (df), partielles Etaquadrat ( $\eta p 2), N=33$

\begin{tabular}{|c|c|c|c|c|c|c|c|c|}
\hline Test & Kennwert & Effekt & $d f$ & $\begin{array}{c}\text { Quadratischer } \\
\text { Mittelwert }\end{array}$ & $F$ & $p$-Wert & $\begin{array}{c}\text { adjustierter } \\
p \text {-Wert }\end{array}$ & $\eta p 2$ \\
\hline \multirow{2}{*}{ Plantarflexion } & $\begin{array}{c}\text { Maximales isometrisches } \\
\text { Drehmoment }(M x)\end{array}$ & \multirow{4}{*}{ Bein } & 1 & 19644,9 & 36,8 & 0,000 & 0,000 & 0,55 \\
\hline & $\begin{array}{c}\text { Ermüdungsindex } \\
(M 60 / M x)\end{array}$ & & 1 & 0,3 & 13,0 & 0,001 & 0,005 & 0,33 \\
\hline \multirow[t]{2}{*}{ Dorsalflexion } & $\begin{array}{l}\text { Mittleres maximales } \\
\text { isometrisches } \\
\text { Drehmoment }(M 60)\end{array}$ & & 1 & 227,7 & 43,1 & 0,000 & 0,000 & 0,65 \\
\hline & $\begin{array}{l}\text { Ermüdungsindex } \\
(M 60 / M x)\end{array}$ & & 1 & 0,0 & 8,0 & 0,008 & 0,048 & 0,24 \\
\hline \multirow{2}{*}{$\begin{array}{c}\text { Plantarflexion } \\
\text { vs. } \\
\text { Dorsalflexion }\end{array}$} & $\begin{array}{l}\text { Ermüdungsindex } \\
(M 60 / M x) \text { links }\end{array}$ & \multirow{2}{*}{ Ermüdung } & 1 & 0,2 & 18,9 & 0,000 & 0,000 & 0,38 \\
\hline & $\begin{array}{l}\text { Ermüdungsindex } \\
(M 60 / M x) \text { rechts }\end{array}$ & & 1 & 1,0 & 34,6 & 0,000 & 0,000 & 0,57 \\
\hline \multirow{8}{*}{ Laufband } & $\begin{array}{l}\text { Druckmaximum unter der } \\
\text { Ferse }\end{array}$ & Bein & 1 & 305,3 & 4,8 & 0,036 & 0,278 & 0,13 \\
\hline & $\begin{array}{l}\text { Druckmaximum unter der } \\
\text { Ferse }\end{array}$ & \multirow{2}{*}{ Ermüdung } & 1 & 917,3 & 16,7 & 0,000 & 0,003 & 0,39 \\
\hline & $\begin{array}{c}\text { Druckmaximum unter } \\
\text { dem Vorfu } \beta\end{array}$ & & 1 & 100,8 & 5,9 & 0,021 & 0,169 & 0,19 \\
\hline & $\begin{array}{l}\text { Druckmaximum unter der } \\
\text { Ferse }\end{array}$ & \multirow{5}{*}{ Laufgeschwindigkeit } & 2 & 1210,0 & 64,8 & 0,000 & 0,000 & 0,71 \\
\hline & $\begin{array}{c}\text { Druckmaximum unter } \\
\text { dem Vorfu } \beta\end{array}$ & & 2 & 681,6 & 90,4 & 0,000 & 0,000 & 0,76 \\
\hline & $\begin{array}{l}\text { Druckmaximum unter } \\
\text { dem Mittelfu } \beta\end{array}$ & & 2 & 120,8 & 7,9 & 0,001 & 0,007 & 0,21 \\
\hline & Schrittfrequenz & & 2 & 4475,7 & $\begin{array}{c}109 \\
8\end{array}$ & 0,000 & 0,000 & 0,79 \\
\hline & Doppelschrittlänge & & 2 & 37975,1 & $\begin{array}{c}557, \\
6\end{array}$ & 0,000 & 0,000 & 0,95 \\
\hline
\end{tabular}

Beim Laufen zeigte die Testwiederholung einen signifikanten Effekt auf die Schrittlänge und Schrittfrequenz sowie das plantare Druckmaximum unter der Ferse mit geringer Effektstärke (Tab. 1). Im Test 2 vergrößerte sich die Schrittlänge, wohingegen die Schrittfrequenz und das plantare Druckmaximum unter der Ferse abnahmen (Tab. 3). Die lokale Muskelermüdung wies einen signifikanten Effekt auf das plantare Druckmaximum unter dem Vorfuß und der Ferse mit mittlerer Effektstärke und geringeren maximalen Druckwerten nach Vorermüdung auf (Tab. 1 und 4). Ein signifikanter Effekt des Beines mit geringer Stärke bestand beim ersten Kraftmaximum und dem plantaren Druckmaximum unter der Ferse. Das rechte Bein wies höhere Werte des ersten Kraftmaximums und das linke Bein höhere maximale Druckwerte unter der Ferse auf. Eine Wechselwirkung Ermüdung*Bein*Test lag für das plantare Druckmaximum unter der Ferse vor (Tab. 1).

Tabelle 2: Vergleich der Mittelwerte (Standardabweichung), Symmetrieindex (SI), N=33

\begin{tabular}{|c|c|c|c|c|}
\hline \multirow{3}{*}{\begin{tabular}{c} 
Muskelgruppe \\
\multirow{3}{*}{ Plantarflexoren }
\end{tabular}} & Bein & $\begin{array}{c}\text { Maximales isometrisches } \\
\text { Drehmoment }\left(M_{x}\right)[\mathrm{Nm}]\end{array}$ & $\begin{array}{c}\text { Mittleres maximales } \\
\text { isokinetisches Drehmoment } \\
\left(M_{60}\right)[\mathrm{Nm}]\end{array}$ & $\begin{array}{c}\text { Ermüdungsindex } \\
\left(M_{60} / M_{x}\right)\end{array}$ \\
\cline { 2 - 5 } & links & $189,7(40,7)^{a}$ & $97,7(27,1)$ & $0,53(0,15)^{a, b}$ \\
\cline { 2 - 5 } & rechts & $164,1(43,5)$ & $93,8(25,2)$ & $0,63(0,19)^{b}$ \\
\hline \multirow{3}{*}{ Dorsalflexoren } & links & $-21,3(19,8)$ & $-6,9(23,7)$ & $0,39(0,11)^{a}$ \\
\cline { 2 - 5 } & rechts & $37,1(7,3)$ & $14,5(3,9)^{a}$ & $0,37(0,15)$ \\
\cline { 2 - 5 } & SI [\%] & $36,4(13,2)$ & $13,6(3,3)$ & $-17,95(27,37)$ \\
\hline
\end{tabular}

${ }^{a}$ Haupteffekt Bein, Vergleich linkes vs. rechtes Bein, , ${ }^{b}$ Haupteffekt Ermüdung, Vergleich Ermüdungsindex Plantarflexoren vs. Dorsalflexoren links und rechts 
Mit der Laufgeschwindigkeit erhöhten sich sowohl die Schrittfrequenz, die Schrittlänge und das erste Kraftmaximum als auch die plantaren Druckmaxima unter den drei Fußzonen. Ein signifikanter Effekt der Laufgeschwindigkeit bestand für die Schrittlänge mit hoher, für die Schrittfrequenz mit mittlerer und für das plantare Druckmaximum unter dem Vorfuß mit geringer Effektstärke.

Tabelle 3: Vergleich der Mittelwerte (Standardabweichung), Symmetrieindex (SI), N=33

\begin{tabular}{|c|c|c|c|c|c|}
\hline \multirow{2}{*}{ Test } & \multirow{2}{*}{$\begin{array}{c}\text { Laufgeschwindigkeit } \\
{[\mathrm{km} / \mathrm{h}]}\end{array}$} & \multirow{2}{*}{ Bein } & \multicolumn{3}{|c|}{ Druckmaximum $\left[N / \mathrm{cm}^{2}\right]$} \\
\hline & & & Vorfu $\beta$ & Mittelfu $\beta$ & Ferse \\
\hline \multirow{3}{*}{ Baseline } & \multirow{6}{*}{11} & links & $43,1(11)^{c, d}$ & $24,5(5,7)^{d}$ & $46,7(13,6)^{a, c, d}$ \\
\hline & & rechts & $43,2(5,5)^{c, d}$ & $24,5(6)^{d}$ & $46,7(13,7)^{c, d}$ \\
\hline & & SI [\%] & $1(17,5)$ & $1,2(18,5)$ & $-1,8(9,3)$ \\
\hline \multirow{3}{*}{ Ermüdung } & & links & $41,3(9,2)$ & $24,2(6,1)$ & $44(15,4)^{a}$ \\
\hline & & rechts & $41,4(7)$ & $23,8(6,5)$ & $42,3(13,7)$ \\
\hline & & SI [\%] & $1,2(18,9)$ & $-2,3(20,4)$ & $-6(12,5)$ \\
\hline \multirow{3}{*}{ Baseline } & \multirow{6}{*}{13} & links & $45,9(11,3)^{c}$ & $24,9(6,9)$ & $51,6(13,9)^{a, c}$ \\
\hline & & rechts & $45,6(9,6)^{c}$ & $25(7,7)$ & $47,3(14,7)^{c}$ \\
\hline & & SI [\%] & $0,6(18)$ & $1,8(20,4)$ & $-5,2(8,9)$ \\
\hline \multirow{3}{*}{ Ermüdung } & & links & $44,3(9,1)$ & $24,7(6,2)$ & $47,2(15,9)$ \\
\hline & & rechts & $44,1(7,2)$ & $23,9(6,1)$ & $46,1(13,7)$ \\
\hline & & $S I[\%]$ & $O(17,1)$ & $-2,5(18,7)$ & $-1,8(12,7)$ \\
\hline \multirow{3}{*}{ Baseline } & \multirow{6}{*}{15} & links & $45,5(11,1)^{c}$ & $26(7,8)$ & $53,7(16)^{a, c}$ \\
\hline & & rechts & $46,3(8)^{c}$ & $25,7(9)$ & $51,9(16,7)^{c}$ \\
\hline & & SI [\%] & $0,5(16,8)$ & $-0,1(27)$ & $-4,6(12,9)$ \\
\hline \multirow{3}{*}{ Ermüdung } & & links & $46,5(8,9)$ & $25,7(5)$ & $50,4(16)$ \\
\hline & & rechts & $46,5(7,7)$ & $25,3(5,6)$ & $47,8(15,8)$ \\
\hline & & SI [\%] & $O(15,7)$ & $-1,2(22,5)$ & $-4(17,5)$ \\
\hline
\end{tabular}

${ }^{a}$ Haupteffekt Bein, Vergleich linkes vs. rechtes Bein, ${ }^{\mathrm{c}}$ Haupteffekt Ermüdung, Baseline vs. Ermüdung, ${ }^{\mathrm{d}}$ Haupteffekt Laufgeschwindigkeit, 11 vs. 13 vs. 15

\begin{tabular}{|c|c|c|c|}
\hline Test & $\begin{array}{l}\text { Laufgeschwindigkeit } \\
{[\mathrm{km} / \mathrm{h}]}\end{array}$ & Schrittfrequenz [1/min] & Doppelschrittlänge $[\mathrm{cm}]$ \\
\hline Baseline & \multirow{3}{*}{11} & $172,8(12,4)^{d}$ & $215(11)^{d}$ \\
\hline Ermüdung links & & $175,8(12,8)$ & $214(13)$ \\
\hline Ermüdung rechts & & $174,0(13,3)$ & $216(22)$ \\
\hline Baseline & \multirow{3}{*}{13} & $181,4(13,5)$ & $256(17)$ \\
\hline Ermüdung links & & $183,1(13,7)$ & $257(16)$ \\
\hline Ermüdung rechts & & $184,6(12)$ & $259(16)$ \\
\hline Baseline & \multirow{3}{*}{15} & $191,7(12,7)$ & $272(16)$ \\
\hline Ermüdung links & & $191,9(13,5)$ & $274(18)$ \\
\hline Ermüdung rechts & & $191,2(14,4)$ & $273(21)$ \\
\hline
\end{tabular}

\footnotetext{
${ }^{d}$ Haupteffekt Laufgeschwindigkeit,11 vs. 13 vs. 15
} 


\section{Diskussion}

Die Ergebnisse der Krafttests ergaben wie hypothetisch angenommen eine weniger starke lokale Ermüdung der Plantarflexoren vs. Dorsalflexoren mit einer sich einstellenden muskulären Dybalance. Im Vergleich zu anderen Studien (Christina et al., 2001) war die induzierte Muskelermüdung der Dorsalflexoren größer. Die Ursachen dafür liegen in der höheren Kraft der Plantarflexoren aufgrund ihrer größeren Muskelmasse, der abweichenden Funktion beim Laufen, Gehen sowie anderen Alltagsbewegungen, die eine stärkere Beanspruchung der Plantarflexoren induzieren und dadurch deren Ermüdungswiderstandsfähigkeit trainieren. Wie bereits in EMG-Analysen gezeigt (Mizrahi et al., 2000), veränderte sich das arthromuskuläre Verhältnis für die Kontrolle der Fußbewegung mit Konsequenzen für das Abrollverhalten sowie die plantare Druckverteilung. Eine muskuläre Dysbalance im Fußgelenk infolge lokaler Muskelermüdung wurde auch von Christina et al. (2001) für die Dorsalflexoren sowie von Kellis und Lissou (2009) für die Plantar- und Dorsalflexoren beschrieben und kann das Verletzungsrisiko erhöhen (Gerlach et al., 2005; van Mechelen, 1992).

Nach der lokalen Muskelermüdung wurden eine Reduktion des ersten Kraftmaximums und der plantaren Druckwerte unter dem Vorfuß sowie unter der Ferse festgestellt. Diese Ergebnisse stimmen mit Befunden anderer Studien überein (Alfuth \& Rosenbaum, 2011; Bisiaux \& Moretto, 2008; Garcia-Perez et al., 2013; Weist et al., 2004; Willems et al., 2012; Willson \& Kernozek, 1999) überein. Die geringeren plantaren Druckwerte stützen die Hypothese einer möglichen Schutzstrategie, um bei Ermüdung Verletzungen entgegenzuwirken (Zadpoor \& Nikooyan, 2012). Dabei könnten geringere Landekräfte infolge einer reduzierten Vertikalbewegung des Körperschwerpunktes, einer besseren Schockabsorption durch stärkere Beugung im Kniegelenk (Kellis \& Liassou, 2009) und eines flachen Fußaufsatzes verursacht durch ermüdete Dorsalflexoren (Christina et al., 2001) die Reduktion des erstes Kraftmaximums sowie der maximalen Druckwerte unter der Ferse erklären. Dazu passt auch die verringerte Vorfußbelastung, die bei gleicher Laufgeschwindigkeit und reduzierter Kraftfähigkeit der Plantarflexoren auf eine veränderte Kraftrichtung beim Fußabdruck mehr horizontal hinweist. Die Unterschiede im ersten passiven Kraftmaximum mit geringeren Werten des dominanten Beines stützen zudem die Vermutung eines aktiven, flachen Fußaufsatzes mit erhöhter Schockabsorption dieses Beines, zumal kein Unterschied in der Schrittlänge im Vergleich beider Beine festgestellt wurde.

Nach lokaler Muskelermüdung wurde bei gleicher Laufgeschwindigkeit keine Änderung der Schrittfrequenz und Schrittlänge gefunden, das stimmt mit Befunden von Alfuth und Rosenbaum (2011) überein, die ebenfalls keinen Wechsel der Schrittfrequenz ermittelten. Andere Studien gehen dagegen von einer Veränderung der Schrittlänge und Schrittfrequenz nach Ermüdung (Kellis, Zafeiridis, \& Amiridis, 2011) aus. Dabei wurde über eine Reduktion der Schrittfrequenz und einen Anstieg der Schrittlänge (Garcia-Perez et al., 2013; Gerlach et al., 2005) bzw. über einen Anstieg der Schrittfrequenz (Willson \& Kernozek, 1999) berichtet.

Die Asymmetrie in den Kraftvoraussetzungen der untersuchten Badminton Spieler mit höheren Werten des linken Beines sowohl der isometrischen Maximalkraft als auch der isokinetischen Kraftausdauer ist auf dessen Dominanz als Sprungbein zurückzuführen (Van der Harst et al., 2007). Die stärkere Ermüdung der Dorsalflexoren des rechten Beines kann aus einer insgesamt geringeren Kraftfähigkeit des nicht-dominanten Beines begründet werden. Die stärkere relative Ermüdung der Plantarflexoren des dominanten linken Beines erklärt sich dagegen durch die Index-Bildung aus einer höheren Maximalkraft im nicht ermüdeten Zustand, denn auch nach dem Ermüdungsprotoll war die absolute Kraftfähigkeit dieses Beines höher.

Die in den Krafttests festgestellte Beinasymmetrie schlug sich in den Werten des ersten Kraftmaximums sowie dem maximalen Druck unter der Ferse, jedoch nicht in weiteren Messwerten der plantaren Druckverteilung, der Schrittlänge sowie den dazugehörigen Symmetrieindex nieder. Diese Differenzen wurden sowohl in der Baseline als auch unter lokaler Muskelermüdung gefunden. Das deckt sich mit Befunden von Brown et al. (2013), die ebenfalls keinen Zusammenhang zwischen der Ermüdung und der Beindominanz beim Laufen ermittelten. Daraus schlussfolgerten sie, dass die 
Seitendifferenzen beim ermüdeten Laufen wahrscheinlich keinen Bezug zur Beindominanz besitzen, was die vorliegenden Ergebnisse stützen.

Bei der homogenen Badminton Spieler Gruppe beeinflusste die Wechselwirkung Test*Bein*Ermüdung die plantaren Druckwerte unter der Ferse, aber die Belastung unter der Ferse wurde von diesen Faktoren wechselseitig beeinflusst.

In den drei untersuchten Geschwindigkeitsstufen zeigte sich in Übereinstimmung mit der Hypothese ein vergleichbarer Einfluss der lokalen Muskelermüdung auf die plantaren Druckwerte unter den drei Fußzonen, die Schrittlänge und -frequenz, obwohl sich mit Zunahme der Laufgeschwindigkeit die Schrittlänge und Schrittfrequenz sowie die Druckwerte insgesamt und insbesondere unter dem Vorfuß erhöhten.

Die Laboruntersuchungen auf dem Laufband ermöglichen die Kontrolle der Laufgeschwindigkeit und erleichtern die Messung der plantaren Druckverteilung. Demgegenüber steht der Nachteil der ungewohnten Bedingungen mit Beeinflussung der Laufbewegung durch das Laufband. Die im Vergleich von Test 1 und Test 2 registrierte Zunahme der Schrittlänge und Reduktion der Schrittfrequenz bei gleicher Laufgeschwindigkeit kann auf ein noch nicht ausreichendes Gewöhnen an das Laufband zurückgeführt werden. Die separate Gewöhnungseinheit von ca. 30 Minuten sowie das zehnminütige Einlaufen vor den beiden Tests waren für eine Anpassung der Schrittlänge und frequenz nicht ausreichend. Bei gegebener Laufgeschwindigkeit reduzierte sich mit zunehmender Routine die Schrittfrequenz und gleichzeitig vergrößerte sich die Schrittlänge im Test 2. In folgenden Untersuchungen muss mehr Zeit für die Gewöhnung der Lauftechnik an das Laufband eingeplant werden. Eine weitere Limitierung bestand in der plantaren Druckmessung, die nur die vertikale Kraft mit geringerer Messgenauigkeit als die Bodenreaktionskraft mit Kraftmessplatten registriert, aber dafür mehrere Laufzyklen über $30 \mathrm{~s}$ mittelt. Durch Verwendung der Herstellersoftware erfolgte eine Reduktion auf drei Fußzonen, ohne Separierung der Zehen und einer Unterscheidung zwischen medialem und lateralem Fuß, was die Vergleichbarkeit mit anderen Studien einschränkte. Die Auswertung im Krafttest konzentrierte sich auf das jeweilige Maximum ohne das mittlere Drehmoment, die Arbeits- und Leistungswerte zu berücksichtigen. Zudem wurde nur eine Winkelgeschwindigkeit $(60 \%$ ) in einer Positionierung (sitzend) getestet.

\section{Schlussfolgerung}

Wie hypothetisch angenommen, konnte gezeigt werden, dass ein lokales Ermüdungsprotokoll mit gleicher Wiederholungszahl die muskuläre Leistungsfähigkeit der Dorsalflexoren stärker als die der Plantarflexoren herabsetzt und dadurch eine muskuläre Dysbalance im Fuß resultiert, was auf ein erhöhtes Verletzungsrisiko hinweist. Bei Badminton Spieler verringern sich infolge der lokalen Muskelermüdung das erste passive Kraftmaximum sowie die plantaren Druckwerte unter der Ferse und dem Vorfuß bei gegebener Laufgeschwindigkeit im Bereich von 11-15 km/h. Die höheren Kraftfähigkeiten des dominanten Beines waren mit einem geringen ersten passiven Kraftmaximum sowie mit höheren plantaren Druckwerten unter der Ferse sowohl beim Laufen ohne als auch nach lokaler Muskelermüdung verbunden. Bei Laufbanduntersuchungen muss ausreichend Zeit für die Gewöhnung an das Gerät eingeplant werden, weil sonst mit erhöhter Schrittfrequenz und reduzierte Schrittlänge gelaufen wird. Zur Verletzungsprävention sollte ergänzend zum Laufen Krafttraining der Fußmuskulatur mit einem besonderen Schwerpunkt auf die weniger leistungsfähigen Dorsalflexoren integriert werden.

\section{Literatur}

1- Alfuth, M., \& Rosenbaum, D. (2011). Long distance running and acute effects on plantar foot sensitivity and plantar foot loading. Neuroscience Letters, 503 (1), 58-62.

2- Baker, J., Burnstein. A., \& Frankel. V. H. (1972). Fatigue fractures: Biomechanical considerations. Journal of Bone and Joint Surgery, 54, 1345.

3- Bisiaux, M., \& Moretto, P. (2008). The effects of fatigue on plantar pressure distribution in walking. Gait \& posture, 28(4), 693-698. 
4- Brown, A. M., Zifchock, R. A., \& Hillstrom, H. J. (2014). The effects of limb dominance and fatigue on running biomechanics. Gait \& posture, 39(3), 915-919.

5- Burr, D. B. (1997). Bone exercise and stress fractures. Exercise and Sport Sciences Reviews, 25, 171-194.

6- Christina, K. A., White, S. C., \& Gilchrist, L. A. (2001). Effect of localized muscle fatigue on vertical ground reaction forces and ankle joint motion during running. Human movement science, 20(3), 257-276.

7- Cohen, J. (1992). Statistic a power primer. Psychology Bulletin, 155-159.

8- Dickinson. J. A., Cook, S. D., \& Leinhardt. T. M. (1985). The measurement of shock waves following heel strike while running. Journal of Biomechanics, 18, 415-422.

9- Fellin, R. E., Manal, K., \& Davis, I. S. (2010). Comparison of lower extremity kinematic curves during overground and treadmill running. Journal of applied biomechanics, 26(4), 407.

11- Fredericson, M., \& Misra, A. K. (2007). Epidemiology and aetiology of marathon running injuries. Sports Medicine, 37(4-5), 437-439.

12- García-Pérez, J. A., Pérez-Soriano, P., Llana, S., Martínez-Nova, A., \& Sánchez-Zuriaga, D. (2013). Effect of overground vs treadmill running on plantar pressure: Influence of fatigue. Gait \& posture, 38(4), 929-933.

13- Gerlach, K. E., White, S. C., Burton, H. W., Dorn, J. M., Leddy, J. J., \& Horvath, P. J. (2005). Kinetic changes with fatigue and relationship to injury in female runners. Medicine and science in sports and exercise, 37(4), 657-663.

14- Girard, O., Millet, G., Slawinski, J., Racinais, S., \& Micallef, J. P. (2010). Changes in leg-spring behavior during a $5000 \mathrm{~m}$ self-paced run in differently trained athletes. Science \& Sports, 25(2), 99-102.

15- Grimston, S. K., \& Zernicke, R. F. (1993). Exercise-Related Stress Responses in Bone. Journal of Applied Biomechanics, 9(1).

16- Hamill, J., Bates, B. T., \& Knutzen, K. M. (1984). Ground reaction force symmetry during walking and running. Research Quarterly for Exercise and Sport, 55(3), 289-293.

17- Hong, Y., Wang, L., Li, J. X., \& Zhou, J. H. (2012). Comparison of plantar loads during treadmill and overground running. Journal of Science and Medicine in Sport, 15(6), 554-560.

18- Hreljac, A. (2005). Etiology, prevention, and early intervention of overuse injuries in runners: a biomechanical perspective. Physical medicine and rehabilitation clinics of North America, 16(3), 651-667.

19- Hunter, I., \& Smith, G. A. (2007). Preferred and optimal stride frequency, stiffness and economy: changes with fatigue during a 1-h high-intensity run. European journal of applied physiology, 100(6), 653-661.

20- Kellis, E., \& Liassou, C. (2009). The effect of selective muscle fatigue on sagittal lower limb kinematics and muscle activity during level running. journal of orthopaedic \& sports physical therapy, 39(3), 210-220.

21- Kellis, E., Zafeiridis, A., \& Amiridis, I. G. (2011). Muscle coactivation before and after the impact phase of running following isokinetic fatigue. Journal of athletic training, 46(1), 11.

22- Macera, C. A. (1992). Lower extremity injuries in runners. Sports medicine, 13(1), 50-57.

Marti, B., Vader, J. P., Minder, C. E., \& Abelin, T. (1988). On the epidemiology of running injuries The 1984 Bern Grand-Prix study. The American Journal of Sports Medicine, 16(3), 285-294.

23- Mizrahi, J., Verbitsky, O., Isakov, E., \& Daily, D. (2000). Effect of fatigue on leg kinematics and impact acceleration in long distance running. Human movement science, 19(2), 139-151.

24- Morin, J. B., Samozino, P., \& Millet, G. Y. (2011). Changes in running kinematics, kinetics, and spring-mass behavior over a 24-h run. Med Sci Sports Exerc, 43(5), 829-836.

25- Morin, J. B., Tomazin, K., Edouard, P., \& Millet, G. Y. (2011). Changes in running mechanics and spring-mass behavior induced by a mountain ultra-marathon race. Journal of biomechanics, 44(6), 1104-1107.

26- Nagel, A., Fernholz, F., Kibele, C., \& Rosenbaum, D. (2008). Long distance running increases plantar pressures beneath the metatarsal heads: a barefoot walking investigation of 200 marathon runners. Gait \& posture, 27(1), 152-155.

27- Noakes, T.D. (1991). The Lore of Running. Champaign: Human Kinetics. 
Nummela, A., Rusko, H., \& Mero, A. (1994). EMG activities and ground reaction forces during fatigued and nonfatigued sprinting. Med Sci Sports Exerc; 26(5): 605-609.

28- Nyland, J. A., Shapiro, R., Stine, R. L., Horn, T. S., \& Ireland. M. L. (1994). Relationship of fatigued run and rapid stop to ground reaction forces, lower extremity kinematics, and muscle activation. Journal of Orthopaedic and Sports Physical Therapy, 20, 132-137.

29- Olivier, P. (2013). Effect of invertor/evertor and plantar-/dorsiflexor fatigue on plantar pressure distribution. South African Journal for Research in Sport, Physical Education and Recreation, 35(1) http://www.ajol.info/index.php/sajrs/article/view/88107

30- Quammen, D., Cortes, N., Van Lunen, B. L., Lucci, S., Ringleb, S. I., \& Onate, J. (2012). Two different fatigue protocols and lower extremity motion patterns during a stop-jump task. Journal of athletic training, 47(1), 32.

31- Rabita, G., Slawinski, J., Girard, O., Bignet, F., \& Hausswirth, C. (2011). Spring-mass behavior during exhaustive run at constant velocity in elite triathletes. Medicine and science in sports and exercise, 43(4), 685-692.

32- Reber, L., Perry, J., \& Pink, M. (1993). Muscular control of the ankle in running. The American journal of sports medicine, 21(6), 805-810.

33- Riley, P. O., Dicharry, J., Franz, J. A. S. O. N., Croce, U. D., Wilder, R. P., \& Kerrigan, D. C. (2008). A kinematics and kinetic comparison of overground and treadmill running. Medicine and Science in Sports and Exercise, 40(6), 1093.

34- Robinson, R. O., Herzog, W., \& Nigg, B. M. (1987). Use of force platform variables to quantify the effects of chiropractic manipulation on gait symmetry. Journal of manipulative and physiological therapeutics, 10(4), 172-176.

35- Savelberg, H. H., Vorstenbosch, M. A., Kamman, E. H., van de Weijer, J. G., \& Schambardt, H. C. (1998). Intra-stride belt-speed variation affects treadmill locomotion. Gait \& posture, 7(1), 2634.

36- Schache, A. G., Blanch, P. D., Rath, D. A., Wrigley, T. V., Starr, R., \& Bennell, K. L. (2001). A comparison of overground and treadmill running for measuring the three-dimensional kinematics of the lumbo-pelvic-hip complex. Clinical Biomechanics, 16(8), 667-680.

37- Slawinski, J., Heubert, R., \& Quievre, J. (2008). Changes in spring-mass model parameters and energy cost during track running to exhaustion. J Strength Cond Res; 22(3): 930-936.

38- Van der Harst, J. J., Gokeler, A., \& Hof, A. L. (2007). Leg kinematics and kinetics in landing from a single-leg hop for distance. A comparison between dominant and non-dominant leg. Clinical Biomechanics, 22(6), 674-680.

39- Van Mechelen, W. (1992). Running injuries. Sports Medicine, 14(5), 320-335.

Walter, S. D., Hart, L. E., McIntosh, J. M., \& Sutton, J. R. (1989). The Ontario cohort study of running-related injuries. Archives of internal medicine, 149(11), 2561-2564.

40- Wank, V., Frick, U., \& Schmidtbleicher, D. (1998). Kinematics and electromyography of lower limb muscles in overground and treadmill running. International journal of sports medicine, 19(07), 455-461.

41- Weist, R., Eils, E., \& Rosenbaum, D. (2004). The influence of muscle fatigue on electromyogram and plantar pressure patterns as an explanation for the incidence of metatarsal stress fractures. The American journal of sports medicine, 32(8), 1893-1898.

42- Willems, T. M., De Ridder, R., \& Roosen, P. (2012). The effect of a long-distance run on plantar pressure distribution during running. Gait \& posture, 35(3), 405-409.

43- Willson, J. D., \& Kernozek, T. W. (1999). Plantar loading and cadence alterations with fatigue. Medicine and science in sports and exercise, 31(12), 1828-1833.

44- Zadpoor, A. A., \& Nikooyan, A. A. (2012). The effects of lower-extremity muscle fatigue on the vertical ground reaction force a meta-analysis. Proceedings of the Institution of Mechanical Engineers, Part H: Journal of Engineering in Medicine, 0954411912447021. 